\title{
Inclusión educativa universitaria para personas en condición de discapacidad intelectual y la visión social de las neurociencias (teoría informacional)
}

\author{
PAOLA VÁSQUEZ ESPINOZA" \\ Universidad Peruana de Ciencias Aplicadas (UPC) \\ Recibido el 20-11-2017; primera evaluación el 25-12-2018; segunda evaluación el 14-01-19; \\ tercera evaluación 19-02-2019; aceptado el 20-02-2019 \\ Para Eberth, por quien todo tiene sentido \\ Obviamente, debido a mi discapacidad, necesito ayuda. Pero yo \\ siempre he tratado de superar las limitaciones de mi condición y \\ llevar una vida lo más completa posible. He viajado por todo el \\ mundo, desde la Antártida a la gravedad cero. (Stephen Hawking)
}

\section{RESUMEN}

El presente trabajo busca explicar cómo se viene realizando la inclusión educativa universitaria para una persona en condición de discapacidad intelectual. Se postula que el desarrollo de su formación neocortical es independiente para cada ser humano, por lo que los procesos intraindividuales se ven condicionados por lineamientos de carácter externo. De igual forma, se ha buscado realizar el análisis de la normativa existente en torno al concepto de discapacidad, especialmente en la referida al ámbito de la inclusión educativa universitaria. Por tanto, se ha hecho una investigación sobre la normativa que permitiría visualizar estos aspectos en el Perú, así como la revisión de antecedentes en España y Chile. Igualmente, se

\footnotetext{
* Doctora en Medicina - Neurociencias (UNMSM). Egresada del Doctorado en Literatura Peruana e Hispanoamericana (UNMSM) y egresada del Doctorado en Lingüística (UNMSM). Es magíster en Lingüística (UNMSM). Autora del poemario Sorbete escolar y de los siguientes libros: Lenguaje y Comunicación (2011), Bosque de letras (coautoría, USIL, 2015), Laboratorio de la Palabra (coautoría, USIL, 2017) y Armonía de la escritura (coautoría, USIL, 2019). Docente asociada de la Facultad de Letras y Cs. Humanas de la UNMSM en pre y posgrado; docente del posgrado de la Facultad de Medicina Humana de la UNMSM. Es docente en la Universidad Peruana de Ciencias Aplicadas. Sus líneas de investigación son la psicolingüística, inclusión educativa y las neurociencias. Actualmente, es coordinadora académica en la Dirección de Estudios Generales de la Universidad San Ignacio de Loyola. Correo: paolincita1@gmail.com
} 
propone que una visión social de las neurociencias puede servir para el ansiado proyecto de inclusión educativa.

Palabras clave: discapacidad intelectual, habilidades diferentes, inclusión educativa.

University an inclusive education for persons of a condition intellectual disability and the social vision of neurosciences (Information Theory)

Abstract

This paper explains how a university is utilizing an inclusive education approach to help a student who has a condition of intellectual disability. We propose that neocortical development is different for every individual, which means that internal processes are being conditioned by external influences. By the same token, we have analyzed the current local regulations and norms regarding the concept of disability; in particular in reference to inclusion for students with special needs at the university level. Consequently, a research has been carried out to figure out the regulations that would help us interpret these aspects of especial education in Peru, as well as in Spain and Chile. Likewise, we propose that a closer look at the social aspects of the neurosciences would help create the appropriate space for educational inclusion in Peru.

Keywords: intellectual disability, different abilities, educational inclusion.

\section{Inclusáo educacional universitária para pessoas com deficiência intelectual a partir do aspecto social das neurociências (Teoria Informacional)}

Resumo

O presente trabalho procura explicar como a inclusão educacional universitária foi realizada para uma pessoa com deficiência intelectual. Postula-se que o desenvolvimento do treinamento neocortical é independente para cada ser humano, de modo que os processos intraindividuais são condicionados por lineamentos externos. Da mesma forma, buscou-se realizar a análise dos regulamentos existentes em torno do conceito de deficiência, especialmente no campo da inclusão educacional universitária. Portanto, uma investigação foi feita sobre os regulamentos que permitiriam que esses aspectos fossem vistos no Peru, bem como a verificação de antecedentes na Espanha e no Chile. Da mesma forma, propóe-se que um olhar a partir do aspecto social das neurociências possa atender ao tão aguardado projeto de inclusão educacional.

Palavras-chave: deficiência intelectual, habilidades diferentes, inclusão educacional. 


\section{INTRODUCCIÓN}

La sociedad se encuentra conformada por un grupo de personas que coexisten en un determinado territorio. Por tanto, se establece, para un normal funcionamiento, normas que regulan la situación de dicha realidad. Desde esta perspectiva, la educación es la encargada de hacer prevalecer estos lineamientos para que la situación funcione de una manera natural. No obstante, cuando una persona sufre algún tipo de conducta que resulta «extrańa» y hasta amenazante para el resto de miembros de dicha comunidad, entonces debe aparecer una forma diferente de poder «normalizar» algunos procesos.

Desde tiempos muy remotos, las personas se han preguntado ¿qué es lo que resulta normal en una sociedad? Frente a ello, se afirma lo siguiente:

La sociedad humana no está formada por un núcleo duro de normalidad al que le salen abscesos laterales, imperfectos e inadecuados. La sociedad humana es un entramado complejo compuesto de hombres, mujeres, jóvenes, viejos, sanos, enfermos, de distintas razas, orientaciones sexuales, particularidades físicas, etc. Ese heterogéneo paisaje es la normalidad. Todos, igualmente, seres humanos. Y todos igualmente dotados de derechos, aunque las necesidades de todos no sean idénticas (Fernández, 2003, p. 29).

La definición de discapacidad, durante las últimas décadas, ha cambiado y ha pasado de ser una situación intraindividual a considerar aspectos externos. Actualmente, ya no se concibe solamente el apoyo de la sociedad a la persona en condición de discapacidad intelectual, sino que se conceptúa en relación a cuánto el individuo beneficia a la sociedad, a través del descubrimiento de sus capacidades diferentes y absolutamente necesarias. Desde tales perspectivas, se considera lo siguiente: «la discapacidad es el resultado de la incapacidad de las sociedades de ser incluyentes y dar cabida a las diferencias individuales. Son las sociedades, no el individuo, las que deben cambiar, y la Convención proporciona una hoja de ruta para ese cambio» (ONU, 2015, p. 16).

Asimismo, Victoria (2013) menciona que la vida de una persona en condición de discapacidad tiene la misma valoración que el de una persona sin discapacidad. Por tanto, el enfoque remarca que ellas podrían aportar mucho a la sociedad, siempre y cuando sean reconocidas tal y como son.

La situación se establece dado que la investigación al respecto ha permitido observar que la interacción social de una persona en condición de discapacidad genera algún tipo de problema, no porque ella en esencia represente el conflicto, sino que este se origina por la actitud del medio y la falta de adecuaciones necesarias, al no ser capaces de aceptar las diferencias. 
La Asociación Americana sobre Discapacidad Intelectual y Discapacidades del Desarrollo (AAIDD), desde el año 1992, postuló cambios en referencia a la concepción de las personas con transtorno del desarrollo intelectual (TDI), incluyendo presupuestos que evidencien que se fuera más allá de lo psicométrico y se estipulara un cambio de paradigma definido en relación con el individuo en interacción con el contexto. Pero no fue hasta el año 2002 en que el basamento de su postura se trasladó a evidenciar que el retraso mental no era una categoría diagnóstica exclusiva, sino un estado de funcionamiento de la persona (Verdugo, 2003).

El significado de discapacidad ya venía desde el año 2002, a través del cual se debían evidenciar los aspectos enunciados a continuación:

Las limitaciones en el funcionamiento presente deben considerarse en el contexto de ambientes comunitarios típicos de los iguales en edad y cultura. (Asimismo) una evaluación válida ha de tener en cuenta la diversidad cultural y lingüística, así como las diferencias en comunicación y en aspectos sensoriales, motores y comportamentales. (Además) en un individuo, las limitaciones a menudo coexisten con capacidades, (por lo que) un propósito importante de describir limitaciones es el desarrollar un perfil de los apoyos necesarios. (Por último) si se ofrecen los apoyos personalizados apropiados durante un período prolongado, el funcionamiento en la vida de la persona con retraso mental generalmente mejorará (Luckasson, 2002, p. 36).

A partir del año 2008, cuando el concepto de retardo mental cambia por el de discapacidad intelectual, inscrita en la categoría de neurodesarrollo, y desde donde se inscriben diferencias en relación a que las limitaciones en el funcionamiento presente se deben a una concepción mucho más holística, se puede evidenciar un cambio en la mirada general. Por tanto, se enuncia lo siguiente: "para comprender (a) el funcionamiento individual de una persona con discapacidad intelectual dentro de un contexto más amplio y (b) la definición constitutiva que subyace al término discapacidad intelectual, todas las dimensiones del funcionamiento y factores con impacto son importantes» (Wehmeyer, 2008 p. 18).

Es, desde esta perspectiva, que se puede concebir que el desarrollo de una persona en condición de discapacidad intelectual necesita de la concurrencia de diversos factores que permitan aclarar el panorama social en su dimensión exacta. Por todo lo dicho, entonces, se postula que el trastorno del desarrollo intelectual coexiste en un ambiente que lo promociona como una característica negativa de la personalidad, y que incluye las evidencias de las restricciones más que de las oportunidades. Todos tenemos limitaciones y poseemos habilidades diferentes en cualquier área del desarrollo. La única dificultad que promueve 
la sociedad y que deviene desde la época escolástica es una cultura basada en el razonamiento intelectual y que ha valorado un solo lado del encéfalo, el hemisferio izquierdo, lo cual es errado en toda la extensión de la palabra, dado que la verdadera riqueza del hombre gira en torno al desarrollo integral. Este lineamiento promueve que la persona con discapacidad no puede ser medida en términos intelectuales únicamente, sino que de lo que se trata es de evidenciar aspectos relevantes que conciernen a otras esferas, tales como la inteligencia emocional, la intrapersonal, la axiológica, la espiritual, la social, la ecológica, etc., que repercuten en un mundo que necesita mejores personas, íntegras y solidarias. En definitiva, una persona en condición de discapacidad intelectual cumple ampliamente con estos conceptos. Por tanto, de acuerdo a la corriente actual del desarrollo de aprendizaje basado en competencias, una postura como la propuesta adquiere importancia a través de sus conceptos de valor y ética, los cuales aparecen de manera determinante en las personas involucradas en el presente estudio.

Desde esta perspectiva, la discapacidad responde a una actitud del medio para con el individuo, más que el significado que se alcanza desde el punto de vista médico. Vista así la definición, el accionar de la persona con discapacidad incluye al desarrollo sostenible, la inclusión social e igualdad de oportunidades en el marco jurídico de una nación y el Estado, puesto que cualquier forma de discriminación vulnera los derechos fundamentales de los ciudadanos. Frente a tal realidad, la pregunta es si la universidad en el Perú (y nos atrevemos a decir en Latinoamérica) está preparada para realizar una auténtica inclusión con los educandos que presentan dichas capacidades.

Estamos convencidos de que existe un acuerdo tácito acerca de la mirada de inclusión que se pretende evidenciar; sin embargo, todavía nos encontramos un poco lejos del concepto real del término. Consideramos que no se trata de un no querer hacer, sino de una falta de conocimiento real sobre los lineamientos que se requieren para poder abordar el problema. Existen algunos avances, pero todavía resultan insuficientes para establecer que existe un verdadero pacto de igualdad dentro de los miembros de la comunidad.

El punto de vista que se aborda en el presente artículo considera que la discriminación hacia las personas con habilidades diferentes no se trata de una situación que se genere por hechos naturales, sino más bien por una sociedad que todavía no conoce las potencialidades que una persona con discapacidad puede desarrollar. Por tanto, el conocimiento del derecho y los accesos de estas personas se torna un asunto de suma importancia, ya que permitirá su eficiente tratamiento, puesto que los cambios que se deben producir giran en 
torno a la percepción de la mayoría de ciudadanos, debido a que vivimos en una democracia, aunque en muchos casos, esta forma no aborde a las minorías.

Por todo lo enunciado anteriormente, la presente exposición busca explicar el significado de lo que una persona en condición de discapacidad intelectual representa dentro del ámbito universitario, ya que la educación se ha tornado diversificada y responde a las competencias que cada individuo posee, y se sostiene que la formación de una personalidad es independiente para cada ser humano. Se tratará, asimismo, de responder a dicho planteamiento a partir de la visión social de las neurociencias, en relación a la Teoría Informacional. Para ello, por tanto, se enunciarán los antecedentes desarrollados sobre inclusión educativa universitaria en otras latitudes, así como el análisis del marco legal en el Perú, la visión de la Teoría informacional y cómo las neurociencias sociales podrían aportar al desarrollo integral de la persona.

\section{MarCo teórico}

\subsection{Antecedentes}

Dentro del marco de América Latina y Europa, existen algunos avances en relación a la inclusión educativa, especialmente para las personas que se encuentran en condición de discapacidad intelectual. Frente a esta propuesta, se podría evidenciar el caso de la Universidad Autónoma de Madrid (UAM), en donde se viene trabajando, desde hace más de una década, en beneficio de las personas con habilidades diferentes y que al decir de Cerrillo y Egido (2013):

El Programa supone una apuesta decidida por la inclusión de personas con discapacidad intelectual en la Universidad. Entre sus características, destacan las siguientes: se centra en la persona y persigue promover la autodeterminación; requiere el trabajo coordinado de un equipo de formación y un equipo de mediación laboral; permite la participación de estudiantes de Magisterio y Psicopedagogía mediante grupos de aprendizaje cooperativo; incluye un periodo de Prácticum que se desarrolla en distintos servicios y departamentos de la UAM; y, por último, es objeto de investigación continua con el ánimo de mejorar, permanentemente, su configuración curricular y su organización (p. 46).

El estudio da cuenta acerca de un programa aplicado en donde existen logros en cuanto a la inclusión laboral por parte de egresados con las características propuestas. Asimismo, formula una serie de resultados que conducen a un estudio sobre las diversas especificaciones en el ámbito de la mejora de la universidad, vista como una comunidad inclusiva. 
De igual forma, en dicha universidad se toma en consideración a otros autores (Riddell, Tinklin y Wilson, 2005; Ryan y Struths, 2004; Fuller, Healey, Bradley y Hall, 2004), quienes han desarrollado aportes que van desde los cambios en la identidad personal y social de las personas en condición de discapacidad intelectual hasta las transformaciones que se operan en la sociedad.

Otro de los trabajos revisados se encuentra inscrito en el texto de Lissi, Zuzulich, et al. (2013) de la Universidad Católica de Chile, en la que se expone la experiencia recogida por los agentes involucrados en la inclusión educativa a nivel superior y la recolección de estrategias que han venido trabajándose a lo largo de casi una década en diversas experiencias educativas. La idea de este proyecto es la generación del debate en torno a situaciones que han marcado las profundas diferencias entre la universidad inclusiva y la que no lo es.

Como se puede apreciar, la realidad por la que trasunta la inclusión educativa universitaria todavía exige un acompañamiento dentro del ámbito de la correspondencia entre lo que se piensa y lo que realmente existe. Si bien aparecen algunos alcances de manera tangencial que ya se pueden evidenciar en diversas latitudes que así lo demuestran, todavía resulta incompleto para todo el marco necesario.

\subsection{Marco legal}

La universidad es uno de los pocos espacios en donde el conocimiento se puede desarrollar de una forma autónoma. Por ello, se debe recordar la relevancia y poder académico que esta ejerce en la sociedad. No obstante, todavía existe una mirada sesgada sobre los procesos inclusivos en el país. Si bien existen algunos avances sobre el tema, especialmente en cuanto a la normativa, todavía el hecho de no aceptar las diferencias individuales imposibilita mirar al otro como un ser humano con capacidades diferentes, pero no menos válidas. Esta realidad hace que no se pueda hablar de una verdadera educación inclusiva e integradora, que sea viable y de largo alcance para todos aquellos que deseen tener acceso a ella.

Sobre la base de estos conceptos, los avances a nivel de las prácticas inclusivas hacia las personas en situación de discapacidad intelectual, caracterizadas por un déficit en el desarrollo de sus capacidades de tipo intelectual, lingüístico y reflexivo, así como en el pensamiento formal, aún se encuentran en proceso. Por esta razón, la educación superior, para muchos, no resulta ser una opción viable, dado que el costo que demanda las adaptaciones a partir de los ajustes razonables, resultaría muy alto. Por tanto, en el Perú, todavía el sueño de una universidad inclusiva en su esencia no ha conformado criterios determinados. 
En el primer artículo de la Declaración Universal de los Derechos Humanos, se menciona que toda persona debe «nacer libre e igual en dignidad y derechos»; asimismo, el artículo 26 inscribe: «toda persona tiene derecho a la educación»(ONU, 1948, p. 1). La Constitución Política del Perú menciona, en su artículo 1, «la defensa de la persona humana y el respeto de su dignidad son el fin supremo de la sociedad y del Estado"; del mismo modo, en el artículo 13, menciona que: «la educación tiene como finalidad el desarrollo integral de la persona humana» y, en el artículo 15, se propone que "el educando tiene derecho a una formación que respete su identidad, así como al buen trato psicológico y físico» (Constitución Política del Perú, 1993, p. 3).

Por su parte, la Ley Universitaria 30220 (2014) establece, dentro del inciso 4 del artículo, «colaborar de modo eficaz en la afirmación de la democracia, el estado de derecho y la inclusión social» (p. 6). Asimismo, en el artículo 38, se expone que «las universidades, institutos y escuelas superiores, públicos y privados, realizan ajustes razonables para garantizar el acceso y permanencia de la persona con discapacidad, incluida la adecuación de sus procesos de admisión. Estas instituciones reservan el 5\% de las vacantes ofrecidas en sus procesos de admisión por especialidad profesional para la postulación de personas con discapacidad, quienes acceden a estos centros de estudio previa aprobación de la evaluación de ingreso" (p. 6). Por último, en el artículo 129 , se menciona que «las universidades implementan todos los servicios que brindan, considerando la integración a la comunidad universitaria de las personas con discapacidad, de conformidad con la ley 29973, Ley General de la Persona con Discapacidad» (p. 6).

Entonces, si desde el año 1946, en que se promulgaron universalmente los derechos humanos, las personas somos iguales en oportunidades y en dignidad, ¿por qué todavía existen barreras en el aspecto educativo a nivel básico y superior? Esta situación se refuerza con el ambiente de discriminación a través del cual el sujeto con TDI se desenvuelve. En ese sentido, la realidad con la que las personas con TDI interactúan aún se mantiene agreste, a pesar de que, en nuestra constitución, se menciona claramente que la educación tiene como fin el «desarrollo integral de la persona». Así, cabe la pregunta: ¿a qué se denomina desarrollo integral?

Desde aquí, es preciso afirmar que el proceso de inclusión en la universidad obedece a lineamientos que aún no han sido establecidos en todos sus ámbitos, especialmente, los referidos a asuntos de organización y de un análisis exhaustivo de la realidad. Dichas propuestas conllevan problemas de carácter fundamental y de soluciones que se podrían generar si es que se vislumbrara un cambio dentro de los esquemas cognitivos y conductuales de la sociedad 
en su conjunto. Al respecto, el significado que la Unesco propone sobre el tema es:

La inclusión se ve como el proceso de identificar y responder a la diversidad de las necesidades de todos los estudiantes a través de la mayor participación en el aprendizaje, las culturas y las comunidades, y reduciendo la exclusión en la educación. Involucra cambios y modificaciones en contenidos, aproximaciones, estructuras y estrategias, con una visión común que incluye a todos (...) La educación inclusiva es una aproximación de desarrollo a partir de la búsqueda de atender las necesidades de aprendizaje de todos los niños, jóvenes y adultos con especial énfasis en aquellos que son vulnerables a la marginalización y la exclusión (Unesco, 2012, p. 16).

Si observamos este contexto, entonces se puede mencionar que la inclusión está dirigida a cualquier persona, ya que todos poseemos diferentes ritmos y formas de aprender. Sin embargo, la situación se torna complicada cuando nos enfrentamos a individuos que poseen diversas connotaciones o que no responden a lo conocido regularmente. Lo desconocido tiende a generar miedos o reparos, sin comprender a profundidad sobre qué se trata o cuáles son las habilidades diferentes que las personas pueden presentar. Este hecho, en algunos casos, no permite que observemos un auténtico proceso para el logro de las competencias, cuyo concepto determina un desarrollo y logro conforme a las capacidades del sujeto, visto de manera particular, mas no en el marco de una diagramación general, acorde a un sistema impuesto que todos debamos seguir, sin mayores cambios.

De acuerdo con la Convención sobre los Derechos de la Personas con Discapacidad y el Protocolo Facultativo de las Naciones Unidas, se debe considerar los principios propuestos en el año 2006, los cuales se rigen mediante los lineamientos de la Carta de la ONU que proclama la libertad, la justicia y la paz, teniendo como base el valor inherente y los derechos iguales e inalienables de todos los miembros de la familia humana. De igual forma, destaca la necesidad de garantizar el ejercicio pleno de las personas con discapacidad, sin discriminación de ninguna índole. Por tanto, reconoce que la discapacidad es «un concepto que evoluciona y que resulta de la interacción entre las personas con deficiencias y las barreras debidas a la actitud y al entorno que evitan su participación plena y efectiva en la sociedad, en igualdad de condiciones con las demás» (ONU, 2006). El Ministerio de Educación del Perú (2012) sobre el presente tratado expone:

La educación inclusiva aspira a habilitar las escuelas para que sean capaces de acoger y responder a las necesidades de todos sus alumnos y de promover 
una vida en comunidad y de participación; ser un espacio de apertura, que alienta el sentido de pertenencia, que motive la participación activa de todos sus miembros en la vida institucional y favorezca el apoyo individual para desarrollar al máximo las potencialidades de los estudiantes. Este enfoque de la educación reconoce y valora la diversidad en el espacio escolar, la misma que se expresa en los diferentes estilos, ritmos, capacidades y necesidades que los estudiantes tienen frente a su aprendizaje. Lo cual ha significado la transformación progresiva del sistema educativo en sus políticas, culturas y prácticas (p. 15).

Por tanto, se puede afirmar que las personas en situación de discapacidad intelectual, como es el caso de las personas con trastorno del desarrollo intelectual (TDI), son sujetos de derecho como cualquier otro individuo, por el simple hecho de ser personas. Entre dicha equidad, se encuentra el acceso a la educación. Sin embargo, todavía la mirada de la inclusión educativa en el Perú y, de manera particular, en el nivel superior, aún no se encuentra desarrollada. De este modo, se evidencia una suerte de discriminación indirecta para las personas afectadas. Por esa razón, se debe recordar que es importante trabajar con esta población, dado que en la ley 29973, Ley General de la Persona con Discapacidad (2012), se estipula lo siguiente:

38.1 Las universidades, institutos y escuelas superiores, públicos y privados, realizan ajustes razonables para garantizar el acceso y permanencia de la persona con discapacidad, incluida la adecuación de sus procesos de admisión. Estas instituciones reservan el 5\% de las vacantes ofrecidas en sus procesos de admisión por especialidad profesional para la postulación de personas con discapacidad, quienes acceden a estos centros de estudio previa aprobación de la evaluación de ingreso.

38.2 La persona que se vea forzada a interrumpir sus estudios superiores por la adquisición de una discapacidad mantiene su matrícula vigente por un período de hasta cinco años para su reincorporación, incluidos los miembros de las Fuerzas Armadas y de la Policía Nacional del Perú que cursan estudios superiores.

Artículo 39. Formación superior en discapacidad Las universidades, institutos y escuelas superiores, públicos y privados, incluyen asignaturas sobre discapacidad en los currículos y programas para la formación de técnicos y profesionales en los campos de la educación, el derecho, la medicina, la sicología, la administración, la arquitectura, la ingeniería, la economía, la contabilidad y el trabajo social (pp. 16-17).

De igual forma, acorde con la Ley Universitaria 30220 (2014), se establece lo siguiente: 
98.6 Las personas con discapacidad tienen derecho a una reserva del $5 \%$ de las va- cantes ofrecidas en sus procedimientos de admisión.

Artículo 129. Integración de personas con discapacidad en la comunidad universitaria Las universidades implementan todos los servicios que brindan considerando la integración a la comunidad universitaria de las personas con discapacidad, de conformidad con la Ley 29973, Ley General de la Persona con Discapacidad. Y es que, si no se entiende que la finalidad última del ejercicio de los derechos es conseguir la plena autonomía de la persona, entonces no se podrá crear la necesaria institucionalidad jurídica para que las personas con discapacidad puedan disfrutar de sus derechos en igualdad de condiciones que el resto de la sociedad. Por tanto, todos los espacios sociales deberán ser plenamente inclusivos para que todos sus miembros puedan participar (...) En este contexto, la labor de la universidad, como conciencia crítica de la sociedad, se vuelve más necesaria que nunca para reivindicar la personalidad plena de las personas con discapacidad frente a doctrinas que los excluyen de la participación en la sociedad (p. 24).

Descubrir datos sobre los porcentajes de las prácticas inclusivas en el Perú a nivel universitario resulta muy difícil, ya que, si bien en todas las universidades existe un lineamiento que prevé esta circunstancia, también resulta limitado en su aplicación. Es verdad que hay avances referidos a la discapacidad de tipo física y, en alguna medida, sensorial. Sin embargo, el avance en cuanto a aquella discapacidad que se refiere a los componentes de tipo cognitivo y social es muy limitado. Al respecto, Salinas (2014) menciona que «la Universidad del siglo XXI debe ser consciente de este desequilibrio que genera el desarrollo individualista en perjuicio de la sociedad, de esta carencia de relación estrecha entre el individuo y su sociedad. Debe ser misión de la Universidad del siglo XXI de crear un modelo alternativo de desarrollo que tienda el puente entre el individuo y su sociedad» (p. 2).

Ahora, se torna necesario conocer cómo la deficiente aplicación del marco normativo evidencia una falta de conocimiento en relación al desempeño de la persona con discapacidad que es incluida. Estos hechos evidencian que las personas con TDI pueden cumplir diversas capacidades en los ámbitos de la vida académica si se realizan los llamados «ajustes razonables». Esta situación conlleva a poder identificar verdaderas líneas democráticas, en donde la equidad sea lo más importante para el desarrollo sostenible de una nación. De lo que se trata es de poder erradicar gran parte de los asuntos referidos a temas como la pobreza y la accesibilidad en los ámbitos sociales, económicos y culturales, así como de salud y educación. 
Si se considera la perspectiva de la teoría cognitiva y constructivista, se puede mencionar que el ser humano es una persona holística, a través de la cual no se puede hablar de escisiones. Muchas veces se entiende, por un lado, un desarrollo intelectual y, por el otro, el resto de las capacidades, como si se tratara de un organismo aislado. La teoría informacional ofrece caminos para convertir a la persona en un ser «integral y moral» (Ortiz, 2006) y ofrece las bases para desarrollar un plan que podría reducir las diferencias entre lo que «somos y lo que debemos ser» (Ortiz, 2006). No cabe duda que, si se realizaran los grandes cambios necesarios en la universidad para cambiar el paradigma existente en torno a la discapacidad, entonces la mirada del otro sería diferente, dado que se entendería que los procesos sociocinéticos contribuyen al desarrollo del neocórtex. Las personas en situación de discapacidad no son la excepción para esta regla.

Por otra parte, el concepto de competencia se define como una asociación de saberes conceptuales, procedimentales y actitudinales que actúan de manera conjunta y para el logro del resultado se necesita evidenciar cada uno de estos aspectos. Por tanto, el pensamiento de solo potenciar uno de ellos, vale decir el que se refiere a un saber conceptual, haría de la persona un ser desmembrado y que no se sienta realmente parte integrante de una sociedad. Las personas en condición de discapacidad intelectual presentan limitaciones, como todas las que presentamos el resto de individuos. Eso no es óbice para que el «desarrollo integral» del que se ha hablado líneas arriba no pueda darse. De lo que se trata es de hacer las adaptaciones correspondientes al currículo, que sea mejore su formulación y que promueva, a través de una evaluación conjunta, un dominio en lo conceptual, social y práctico, desde la perspectiva médica que se asume en el DSM-V (American Psychiatric Association, 2014).

Por tal razón, no se puede considerar solamente un aspecto de lo que cada individuo representa, sino que se le debe ver desde diversas perspectivas, y si a eso se le aúna que la persona con TDI es un individuo social, entonces cabe la siguiente cita:

De igual manera, producto de recientes investigaciones, se ha descubierto la plasticidad cerebral, la cual es definida por el Instituto de Familia y Trabajo de la Universidad de Chicago como «La capacidad del cerebro para cambiar en respuesta a las demandas del ambiente». Esto indica que las capacidades individuales no están fijadas al momento del nacimiento. El cerebro mismo puede ser alterado o ayudado para compensar problemas y para intervenir en el momento apropiado (Amar, Abello y Tirado, 2005, p. 46).

Ningún diagnóstico presuntivo es determinante únicamente por sí mismo. Existen categorías que pueden presentar las personas; sin embargo, eso no 
determina la discapacidad, dado que esta se encuentra enmarcada a través de lo que la sociedad y sus accesos presumen sobre ella. Frente a este hecho, se debe tomar en consideración que lo que busca el presente ensayo no es que todas las personas que presentan TDI deban ingresar a la universidad, tal como se espera con los sujetos que no presentan discapacidad. El presupuesto que se sostiene es que la persona deba tener la libertad de elegir y las oportunidades de ser tratado con respeto en el marco de su desarrollo integral, que al decir de Ortiz no es otro que:

La transformación moral de la sociedad, puesto que nuestra preocupación es, cómo se puede desarrollar una teoría del hombre que nos permita realizar las grandes aspiraciones éticas, [...] y para ello tenernos que llevar adelante las ideas fundamentales, promoviendo precisamente la solidaridad, la libertad y la justicia. [...] Desde nuestro particular punto de vista, y ojalá desde todo punto de vista, no hay otra finalidad que esté por encima de la formación integral de los hombres, que debe comenzar y terminar con la formación moral de la conciencia (Ortiz, 2002, p. 128).

\subsection{Acerca de la teoría informacional}

A partir de lo enunciado, se debe explicar que la teoría informacional de las neurociencias, desarrollada por el doctor Pedro Ortiz, considera que lo fundamental en cualquier proceso de cambio se encuentra en la doble determinación, la cual expone que lo intraindividual puede ser condicionado a partir de la realidad circundante, lo extraindividual o también llamado procesos sociocinéticos que motivan la formación del neocórtex, y que pueden vislumbrar una auténtica realización para el desarrollo de las competencias en personas con habilidades diferentes. Al respecto, el creador de la teoría informacional nos dice que «la tarea de la ciencia neural es proveer explicaciones del comportamiento en términos de las actividades del cerebro, explicar cómo millones de células nerviosas individuales operan en el cerebro para producir un comportamiento y cómo, a su vez, estas células son influenciadas por el ambiente, incluyendo el comportamiento de otras gentes» (Ortiz, 2006, p. 96).

Las sociedades de tipo multiindividual se convirtieron en sociedades supraindividuales que permitieron los cambios del neocórtex a partir de la información que se almacenó como información genética, tisular, paleocortical y determinó repercusiones a nivel del individuo. Este mismo enunciado adquiere sentido para el trabajo materia de la presente investigación, dado que los cambios que se producen en forma extraindividual van a determinar los procesos de desarrollo de tipo intraindividual. Por tanto, desde esta concepción, 
el accionar de la persona en condición de discapacidad intelectual va a estar condicionada, en gran parte, por las transformaciones que se producen desde afuera del individuo hacia adentro, siempre y cuando se encuentren determinados por una adecuada convivencia, que respete las diferencias y, sobre todo, que sea capaz de identificar que la sociedad en su conjunto es el resultado de una serie de procesos y cambios, en donde todos los organismos son igual de importantes para el desarrollo.

Esta situación es susceptible de ser analizada en virtud de que, en realidades como las del Perú y gran parte de Latinoamérica, donde la inclusión no se produce de manera natural, el proceso resulta mucho más complicado, en especial si se trata de la educación superior. A esto le añadimos que hablar de discapacidad, en términos generales, conllevaría un amplio espectro de posibilidades y la inclusión no es igual en todos los procesos. Por tal razón, este hecho solo podrá ser evidenciado si se propone una real inclusión para las personas que presentan condiciones de discapacidad intelectual o, como lo llama el ámbito médico, el trastorno del desarrollo intelectual (TDI) (American Psychiatric Association, 2014), puesto que estos sujetos, a los que comúnmente se les conocía como personas con retraso mental han sido los que mayor exposición al maltrato han obtenido a lo largo del tiempo. Lo que no se ha analizado es que, si se aborda el tratamiento y ajustes razonables en relación a objetivos, metodología y lineamientos especializados con las personas en condición de discapacidad intelectual, el resultado sería mucho más viable, ya que contaría con un testimonio a partir de un enfoque integral funcional que redundará en la historia de la persona y la ayudará a conformar su sistema sociobiológico.

\subsection{Punto de vista social de las neurociencias (teoría informacional) e inclusión educativa universitaria}

Se ha llegado hasta aquí haciendo un recuento de lo que sucede en el marco legal en relación a la persona que se encuentra en condición de discapacidad intelectual para evidenciar acuerdos en torno a lo que la sociedad y sus accesos nos ofrece, específicamente respecto a la inclusión educativa universitaria. Asimismo, se ha mencionado que existe una doble determinación, a partir de la cual los procesos externos influyen en la formación de la persona: "precisamente tenemos que saber explicar cómo, por qué e inclusive para qué, la sociedad transforma a nuestro cerebro en una conciencia, y cómo esta determina que el cuerpo se transforme en una personalidad» (Ortiz, 2009, p. 208).

Se puede observar que la actividad de la persona se encuentra totalmente organizada. Por un lado, la determinación epigenética, que incluye a los 
procesos internos del individuo; y, por el otro, la determinación sociocinética, que incluye a los procesos extraindividuales (véase anexo 1). Desde la perspectiva de la teoría informacional, ambas situaciones se encuentran íntimamente relacionadas y se influyen mutuamente, partiendo de la conformación genético molecular, hasta el consciente neocortical. En otras palabras, la formación de la persona está influida por la información que recibe del medio, lo que en neurociencias se denomina como la doble determinación (Ortiz, 2012, p. 49). La relación que existe entre la sociedad y el individuo responde a lo que la persona ha recibido y recibe a lo largo de su vida que, al final de cuentas, va a definir la formación de su neocórtex. Por tanto, no se trata de solamente etiquetar a una persona que se encuentra diagnosticada con TDI, con una postura racionalista de los siglos XVI y XVII, mediante la cual muchos aspectos no pueden ser modificados y, sobre todo, aceptados a partir de las diferencias individuales, sino que de lo que se trata es de entender que la persona se ve influenciada por el medio que la rodea. Desde este punto de vista, la relación sociedad-individuo conforma un espacio de ida y vuelta, a través del cual todos se benefician.

La fundamentación de la neurociencia social radica en que esta debe poseer una racionalidad acorde con la estructura de los seres vivos y su relación con la comunidad; por tanto, resulta totalmente necesario que esta ciencia incluya en su proyecto científico a las personas con discapacidad. La teoría del psiquismo, la salud y la educación debe considerar los accesos hacia los cuales se encuentra dirigida realmente la formación de las personas y el consecuente desarrollo de la sociedad. De lo que se trata es de evidenciar que la discriminación se produce por alguna deficiencia de los métodos o los contenidos de las teorías médicas, psicológicas o pedagógicas, y su consecuente derivación del desconocimiento que aún mantenemos los seres humanos (Ortiz, 2006, p. 149).

Este aspecto conlleva a entender que la persona no es producto únicamente de procesos que devengan en aspectos internos, sino que estos se encuentran íntimamente unidos y condicionan la formación de estructuras neocorticales. Si se entiende esta idea como una categoría que observa la conformación orgánica, entonces imaginemos cómo lo funcional puede estar condicionado por una exposición externa al sujeto.

El cambio de paradigma a través del cual se entienda que la sociedad influye en la persona no es nuevo. Los especialistas trabajan al respecto y dan cuenta de este sistema de orden de manera natural. Más allá de las diferencias, lo que hoy nos debe quedar claro es que tal como es la persona, así debe ser aceptada, porque la riqueza del ser humano radica, justamente, en poder definir que cada uno es totalmente diferente al otro, sea que se encuentre en condición de discapacidad o no. 
Erick Kandel, premio Nóbel del año 2000 en medicina, por sus estudios en neurociencias dice lo siguiente: «la tarea de la ciencia neural es proveer explicaciones del comportamiento en términos de las actividades del cerebro, explicar cómo millones de células nerviosas individuales operan en el cerebro para producir un comportamiento, y cómo, a su vez, estas células son influenciadas por el ambiente, incluyendo el comportamiento de otras gentes» (Kandel, Schwartz y Jessell, 2008, p. 258).

Ahora bien, desde la perspectiva que compete al presente trabajo, se debe mencionar que en la formación de la persona se toma en cuenta que:

(La) actividad psíquica personal tiene dos formas de determinación: así, de un lado, el nińo, como todo miembro de la especie Homo Sapiens, nace con una actividad psíquica no consciente, la cual como toda actividad psíquica animal se genera epigenéticamente a partir de la actividad nerviosa, que es la que refleja las condiciones internas del individuo, y se organiza en sólo dos sistemas psíquicos: uno afectivo-emotivo y otro cognitivo-ejecutivo, cuya información, se codifica en la porción más antigua del cerebro que es el alocórtex. Pero, de otro lado, esta actividad psíquica inconsciente — propia de un animal superior- que muestra el nińo al nacer, es punto de partida de su actividad por medio de la cual él empieza a incorporar toda la información social disponible, esto es, sentimientos, conocimientos, valores elaborados a lo largo de la historia de la humanidad. De este modo, esta información social que se asimila e incorpora, se codifica en el neocórtex cerebral, convirtiéndose en información psíquica consciente, y es así como todo el conjunto de esta clase de información llega a constituir lo que llamamos la conciencia personal (Ortiz, 2006 p. 189).

Entonces, se puede afirmar que la persona, sin importar de quién se trate, viene con una actividad paleocortical que forma parte de la información recibida en los niveles genético, tisular, orgánico, pero que terminará de asociar la codificación del neocórtex cerebral a partir de la información social que ha recibido del medio. Esta situación se vislumbra en lo que nosotros consideramos el trabajo que se desarrolla a nivel de las familias o de la comunidad. Asimismo, si poseemos el conocimiento que el neocórtex solamente llega a concluir su maduración aproximadamente a los 22 o 23 años, entonces podemos indicar que la persona que se encuentra en condición de discapacidad intelectual puede verse influenciada (y se ve) por toda la sociedad en su conjunto. La universidad no debería ser extraña a esta situación, puesto que, a la edad propuesta, todavía el sujeto se encuentra en proceso de formación educativa a nivel superior, pero que representa una nueva concepción en los aspectos sociales a los cuales se va a ver expuesto. 
La situación de las personas con TDI condiciona a la sociedad y sus apoyos, tal como lo mencionaba la Asociación Americana sobre Discapacidad Intelectual y Discapacidades del Desarrollo (AAIDD) en el año 2008, la que va a concebir el verdadero cambio y la mejora en las relaciones y en el desarrollo activo. Nuestras universidades podrían ser los centros a partir de los cuales dichos apoyos se brinden en el ámbito educativo (Chiroleu, 2009). La persona con discapacidad coexiste con una serie de capacidades que no se toman en consideración y que deben servir para romper con estereotipos que han acompańado a lo largo del tiempo a las sociedades antiguas y modernas.

De acuerdo con las cifras dadas por el INEI, a través de la Primera Encuesta Nacional sobre Discapacidad (2012), en el Perú, existe un total de un millón quinientos setenta y cinco mil cuatrocientas dos personas con algún tipo de discapacidad, lo que equivale al 5\% de la población. De esta cifra, el 32,1\% presenta dificultades para los procesos cognitivos formales. Por lo que se puede observar, la cantidad resulta representativa y habría que pensar en cuántas de estas personas tienen acceso a la educación o cuántas llegan a la educación superior (INEI, 2014) (Véase anexo 2).

El problema no es tan sencillo, ya que se trata de una visión inclusiva global que deba determinarse como un problema nacional. Sin embargo, esta situación en la realidad no se produce, dado que la única perspectiva desde la que se trabaja radica en el plano médico. El sistema educativo no responde al requerimiento, puesto a que las políticas públicas no permiten los niveles de acceso necesarios para las personas en condición de discapacidad intelectual. Al respecto, la Organización Mundial de la Salud (2003) propone una perspectiva que tenga que ver no solamente con los aspectos médicos, sino sobre todo sociales:

Los factores ambientales constituyen el ambiente físico, social y actitudinal en el que una persona vive y conduce su vida. Estos factores son externos a los individuos y pueden tener una influencia negativa o positiva en el desempeńo/ realización del individuo como miembro de la sociedad, en su capacidad o en sus estructuras y funciones corporales. [...] Los factores contextuales representan el trasfondo total tanto de la vida de un individuo como de su estilo de vida. Incluyen los factores ambientales y los factores personales que pueden tener un impacto en la persona con una condición de salud y sobre la salud y los estados relacionados con la salud de esta persona (p. 19).

Por tanto, los apoyos de tipo familiar y comunitario se hacen imprescindibles para los procesos de inclusión en todos los ámbitos de la vida. La ciencia permite establecer que el medio social puede determinar los niveles de desarrollo para las personas en condición de discapacidad. Por esa razón, el 
trabajo a nivel universitario resulta absolutamente necesario, dado que es en este ámbito desde donde se podrá responder claramente a políticas de inclusión social verdaderas.

\section{Conclusión}

Se ha visto que la discapacidad intelectual, conocida como trastorno del desarrollo intelectual, en su definición, ha cambiado de paradigma, puesto que actualmente se la considera en la relación del individuo con el medio y cómo este puede influir en él. Asimismo, para el caso de nuestro país, la normativa sobre la educación inclusiva en las universidades se encuentra amparada por la Declaración Universal de los Derechos Humanos, la Constitución Política del Perú, la Ley Universitaria (30220), la Ley General de la Persona con Discapacidad (29973), tal como se ha mencionado en líneas anteriores y otros lineamientos de carácter nacional e internacional, por lo que su aplicación resulta totalmente legal y su no aplicación resultaría un proceso ilegítimo, que, lamentablemente, no es parte de una real política de Estado, porque los cambios aplicados resultan insuficientes en cuanto a las necesidades requeridas. Además, la inclusión educativa superior encuentra sustento también en el punto de vista social de las neurociencias, a través del cual la doble determinación del individuo lo hace partícipe de una realidad por la que atraviesa y que resulta necesaria la información social en la conformación del neocórtex. De igual forma, la necesidad de generar reales políticas inclusivas es lo que debe motivar al gran cambio en nuestro país, ya que no solamente se atribuye el concepto desde una postura médica, sino que se resalta la participación comunitaria para el desarrollo de la persona.

Por tal razón, nos atrevemos a decir que todavía nos encontramos lejos de afirmar que la universidad se encuentra preparada para afrontar una inclusión educativa real en lo que respecta a las personas que se encuentran en condición de discapacidad intelectual. Posiblemente, se deba a que la legislación en esta área se enfoca, sobre todo, en el ingreso, mas no en la permanencia y acompañamiento en el transcurso de los años. Lo que en teoría se denomina como los ajustes razonables, todavía se encuentran lejos de ser una realidad. Existen algunos avances en relación a la discapacidad sensorial y física; sin embargo, en cuanto a la discapacidad intelectual, es muy poco es lo avanzado. La situación no es nueva, ya que, en gran parte de América Latina, suceden escenarios semejantes, pero, tal como mencionan los organismos asociados, se trata de empezar a pensar que "(se hace necesario) dejar de considerar las iniciativas en pro de la inclusión como algo que hacemos para ayudar a un grupo que 
necesita apoyo, para pasar a considerarlo como un tema de derecho. Derecho que toda persona tiene a acceder a las oportunidades educativas que existen en nuestra sociedad. Solo entonces podemos empezar a avanzar más rápido hacia la inclusión» (Lissi, Zuzulich et al., 2013, p. 36).

\section{REFERENCIAS BIBLIOGRÁFICAS:}

Amar, J., Abello, R. y Tirado, D. (2005). Efectos de un programa de atención integral a la infancia en el desarrollo de niños de sectores pobres en colombia. Investigación \& Desarrollo, 13(1), 60-77. Recuperado de http:// www.redalyc.org/articulo.oa?id=26813103

American Psychiatric Association (2014). Manual diagnóstico y estadítico de los trastornos mentales DSM-5. Madrid: Editorial Médica Panamericana .

Cerrillo, R. y Egido, I. (2013). Inclusión de jóvenes con discapacidad intelectual en la Universidad. Revista de investigación en educación, 2013, no 11 (1), 41-57. Recuperado el 18 de octubre de 2015, de http://www.researchgate. net/publication/251237810_Inclusin_de_jvenes_con_discapacidad_intelectual_en_la_Universidad

Chiroleu, A. (2009). La inclusión en la educación superior como política pública: tres experiencias en América Latina. Población, 9(3.1), 17-0. Recuperado de http://www.rieoei.org/deloslectores/2740Chiroleu.pdf

Chiroleu, A. (2009). La inclusión en la educación superior como tema de la agenda de gobierno en América Latina. Una reflexión sobre las propuestas del CRES/2008. Universidades, 59(40). Recuperado de http://www.redalyc. org/pdf/373/37313028003.pdf

Fernández, A. (2003). Educación inclusiva:Enseñar y aprender entre la diversidad. Revista digital Umbral, 2-11. Recuperado el 8 de febrero de 2019, de http:// www.inclusioneducativa.org/content/documents/Generalidades.pdf

Fuller, M., Healey, M., Bradley, A. y Hall, T. (2004) Barriers to learning: a systematic study of the experience of disabled students in one university. Studies in Higher Education, 29(3), 303-318. https://doi.org/ 10.1080/03075070410001682592

INEI (marzo de 2014). Primera encuesta nacional especializada sobre discapacidad. Recuperado de http://www.inei.gob.pe/media/MenuRecursivo/ publicaciones_digitales/Est/Lib1 171/ENEDIS\%202012\%20-\%20 COMPLETO.pdf

Kandel, E., Schwartz, J. y Jessell, T. (2008). Principios de neurociencias (Sexta edición en español). Madrid: McGraw-Hill. 
Ley 29973. Ley General de la Persona con Discapacidad (2012) https://www. mimp.gob.pe/webs/mimp/herramientas-recursos-violencia/contenedordgcvg-recursos/conte- nidos/Legislacion/Ley-general-de-la-Persona-conDiscapacidad-29973.pdf

Ley 30220. Ley Universitaria (2014). Fuente: https://www.sunedu.gob.pe/wpcontent/uploads/2017/04/Ley-universitaria-30220.pdf

Lissi, M., Zuzulich, M. et al. (2013). En el camino hacia la educación superior inclusiva en Chile. Santiago, Chile: Universidad Católica de Chile.

Luckasson, R. B.-D. (2002). Mental retardation:definition, classification and systems of support. Washington D.C.: American Association on Mental Retardation.

OMS (2003). Clasificación internacional del funcionamiento, de la discapacidady de la salud. Ginebra, Suiza: Grupo de Clasificación, Evaluación, Encuestas y Terminología. Recuperado de http://www.orientared.com/car/CIDDM-2. pdf

ONU (10 de diciembre de 1948). Declaración Universal de los Derechos Humanos. Recuperado de http://www.un.org/es/sections/what-we-do/ protect-human-rights/

ONU (13 de diciembre de 2006). Convención sobre los Derechos para las personas con discapacidad y protocolo facultativo. Recuperado de http://www.un.org/ disabilities/documents/convention/convoptprot-s.pdf

ONU (12 de agosto de 2015). Oficina del Alto Comisionado para los Derechos Humanos. Recuperado de http://www.ohchr.org/SP/Pages/WelcomePage. aspx

Ortiz, P. (2002). El punto de vista social de la Neurociencia. Recuperado de http:// www.tarpuq.com/sites/default/files/blogPOC/publicaciones/UN\%20 CONCEPTO\%20DE\%20PSIQUISMO.pdf

Ortiz, P. (2006). Formación de la personalidad. Lima: Fondo Editorial de la Universidad Nacional Mayor de San Marcos.

Ortiz, P. (2009). Cuadernos de Psicobiología Social. Introducción a una Psicobiología Social del hombre. Segunda edición. Lima: Fondo Editorial de la Universidad Nacional Mayor de San Marcos.

Ortiz, P. (2012). Lenguaje y habla personal. Lima: Fondo Editorial de la Universidad Nacional Mayor de San Marcos.

Perú (1993). Constitución Política del Perú. Lima: Ministerio de Educación.

Perú (14 de junio de 2012). Ley General de la Persona con Discapacidad 29973. Recuperado de http://www.upch.edu.pe/vracad/oamra/images/pdf/ Ley_29973.pdf 
Perú, G. d. (09 de julio de 2014). Ley Universitaria 30220. El Peruano, XXXI(12914), págs. 527213-527233.

Perú, Ministerio de Educación (marzo de 2012). Educación básica especial y educación inclusiva: balance y perspectivas. Recuperado de http://www.minedu. gob.pe/minedu/archivos/a/002/05-bibliografia-para-ebe/9-educacionbasica-especial-y-educacion-inclusiva-balance-y-perspectivas.pdf

Reglamento de la Ley 29973 (2014) http://evaluaciondocente.perueduca.pe/ media/2016/06/Reglamento_de_la_Ley__de_la_Persona_con_Discapacidad.pdf

Riddell, S., Tinklin, T. y Wilson, A. (2005) Disabled Students in Higher Education, Londres: RoutledgeFalmer.

Ryan, J. y Struths, J. (2004). University education for all? Barriers to full inclusion of students with disabilities in Australian universities. International Journal of Inclusive Education, 8, 73-90. https://doi. org/10.1080/1360311032000139421

Salinas, H. (2014). El rol de la universidad en el siglo XXI. Revista Adital, 86-98. Recuperado el 16 de octubre de 2018 de http://www.unapiquitos.edu. pe/publicaciones/miscelanea/descargas/El_rol_de_la_universidad_en_el_ siglo_XXI.pdf

Unesco (junio de 2012). Inclusión internacional. Recuperado de http://www. inclusioneducativa.org/ise.php?id=1

Verdugo, M. (03 de octubre de 2003). Aportaciones de la definición de retraso mental $(A A M R, 2002)$ a la corriente inclusiva de las personas con discapacidad. Recuperado de http://sid.usal.es/idocs/F8/FDO6569/verdugo.pdf

Victoria, J. (2013). El modelo social de la discapacidad: una cuestión de derechos humanos. Boletin Mexicano de Derecho Comparado, 46(138), 6.

Wehmeyer, M. L. (2008). El constructo de discapacidad intelectual y su relación con el funcionamiento humano. Siglo Cero, 39(227), 5-18. 


\section{ANEXO 1}

NIVELES ESTRUCTURALES DE LA ACTIVIDAD PERSONAL

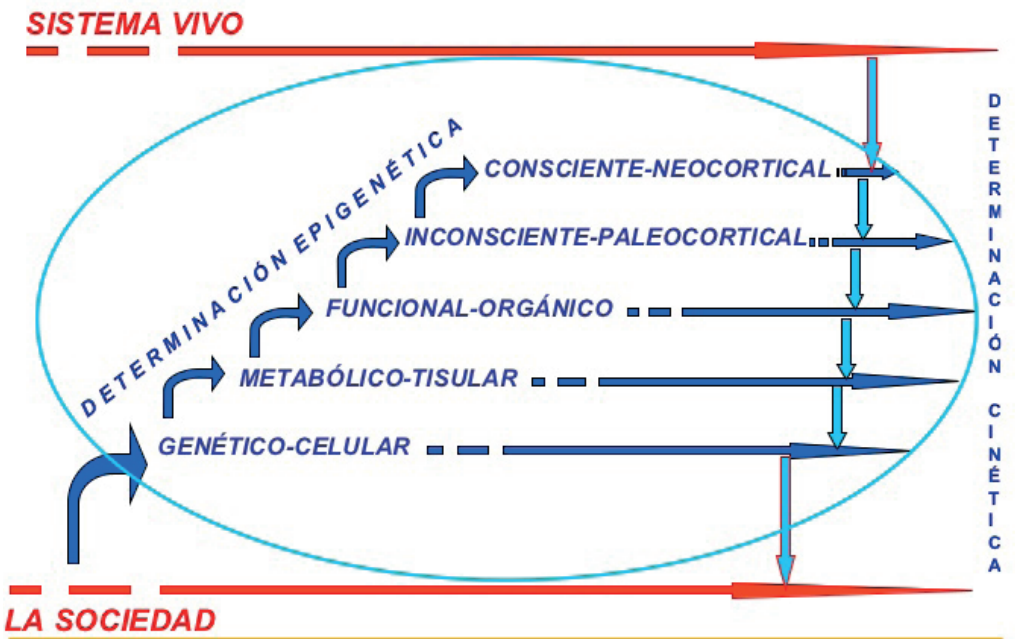

Modificado de: Ortiz, CP. (2004)

\section{LA NEUROCIENCIA SOCIAL DEL CEREBRO PERSONAL EN EL CONTEXTO DE UNA SOCIOBIOLOGÍA DIALÉCTICA}

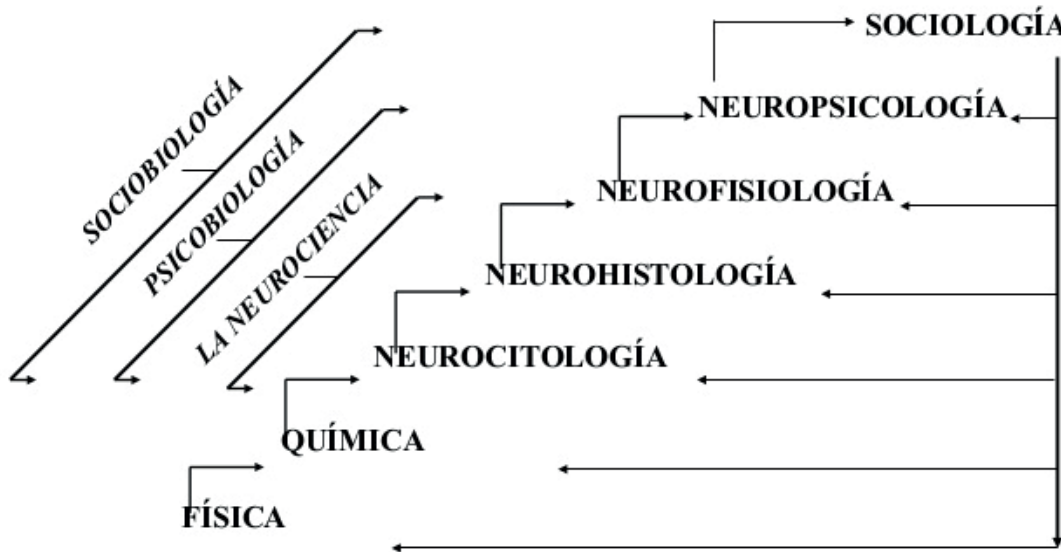

Ortiz CP (2008) 
Inclusión educativa universitaria para personas en condición de discapacidad intelectual

\section{ANEXO 2}

\section{PERÚ: PERSONAS CON DISCAPACIDAD ANALFABETAS DE 15 Y MÁS AÑOS DE EDAD, SEGÚN ASISTENCIA A UN PROGRAMA DE ALFABETIZACIÓN, 2012 \\ (Distribución Porcentual)}

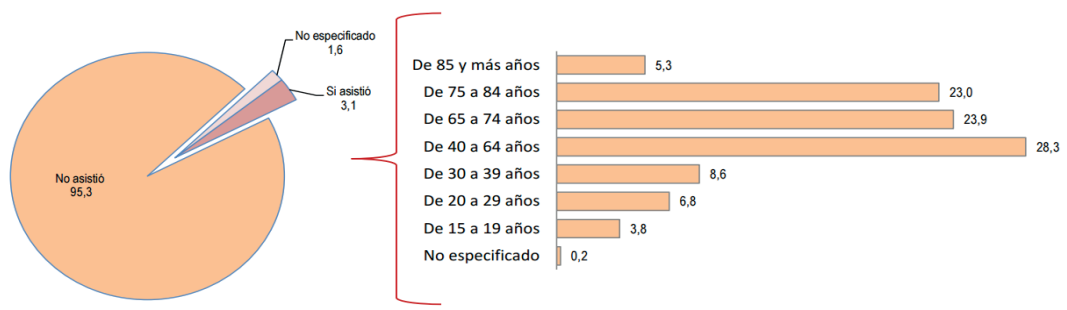

Fuente: Instituto Nacional de Estadistica e Informática - Primera Encuesta Nacional Especializada Sobre Discapacidad 2012.

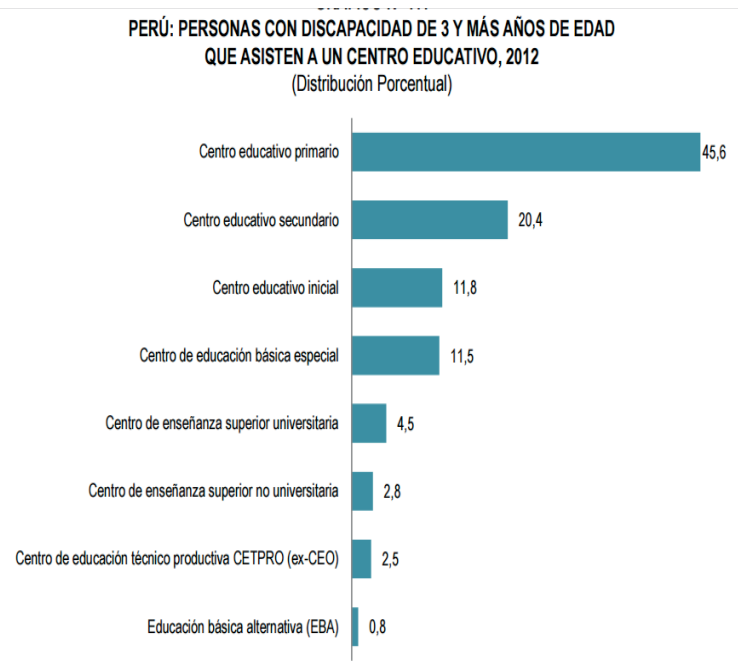

Fuente: Instituto Nacional de Estadistica e Informática - Primera Encuesta Nacional Especializada Sobre Discapacidad 2012. 